\title{
La construcción discursiva de la prevención del delito en México 2006-2009 Miguel Quintana Navarrete*
}

\section{Resumen}

Este estudio analiza la construcción discursiva de la prevención del delito en México durante la primera mitad de la presidencia de Felipe Calderón. Se distinguen las características de diversos sujetos y objetos - así como de las relaciones entre ellos - creados y representados en el discurso oficial preventivo. El análisis revela el sustento axiológico neoconservador de la prevención y el modo en que sus principios son "operacionalizados" con el fin de articularlos a la experiencia cotidiana. Los hallazgos dan sustento empírico a teorías y conceptos criminológicos contemporáneos, principalmente los de "criminologías de uno mismo" y "criminologías del otro", muestran la construcción discursiva de una prevención familiar del delito como un tipo específico de prevención.

\begin{abstract}
This study analyzes the discursive construction of crime prevention in Mexico during the first half of Felipe Calderón's presidency. The characteristics of different subjects and objects - as well as the relations between them - created and represented in the official discourse are distinguished. The analysis reveals the neo-conservative axiological basis of crime prevention, and the way its tenets are "operationalized" in order to articulate them to everyday experience. The findings provide empirical support to contemporary criminological theories and concepts, mainly the notions of "criminologies of the self" and "criminologies of the other", and reveal the discursive construction of a distinct type of crime prevention based on the family.
\end{abstract}

Palabras clave: prevención del delito, análisis de discurso, familia, jóvenes, "criminología de uno mismo" y "criminología del otro".

Key words: crime prevention, discourse analysis, family, young people, "criminologies of the self", "criminologies of the other".

Maestro en Criminología y Justicia Penal por The Dickson Poon School of Law, King's College London. Profesor asociado. Centro de Investigación y Docencia Económicas (CIDE). 
oda teoría criminológica entraña una teoría preventiva del delito: si desde cierta perspectiva se fijan determinadas condiciones como las causantes de la comisión de los ilícitos, es posible idear intervenciones para prevenir la actualización de esas condiciones y, por lo tanto, de la actividad delictiva (Crawford, 1998: cap. 1). Estas teorías adquieren mayor o menor valor explicativo conforme se adecuan a estándares científicos, pero sin lugar a dudas también por la influencia de la organización social, cultural, económica y política de las sociedades en que se presentan (Scheingold, 1998; Melossi 2008).

Existe un consenso en que hacia el último cuarto del siglo XX emergió un nuevo paradigma criminológico conformado por una serie de teorías y formas de pensar sobre el sistema de justicia penal, por lo menos en Estados Unidos y Europa occidental (Feeley y Simon, 1992; Bottoms, 1995; Sparks, 2000; Reiner 2006; Melossi, 2008; O'Malley, 2010). Garland (2001) uno de los académicos más influyentes ha caracterizado esta histórica transformación como la abdicación del programa gubernamental de 'bienestar penal' - basado en la búsqueda de la rehabilitación de los delincuentes y en la expectativa de una solución verdaderamente "social" al problema de la delincuencia - en favor de una "cultura de control" que, entre otras cosas, ha propiciado un papel más activo por parte de la ciudadanía en los temas penales. En el ámbito de la prevención, este cambio se ha traducido en esquemas de alcance comunitario desligados de las grandes reformas sociales de igualdad y eliminación de la pobreza (véase Kemshall, 2003: capítulo 2; Reiner, 2006: 28-29).

En México, el poco trabajo genealógico realizado en relación con las características del sistema de justicia penal en décadas anteriores sugiere que las ideas de resocialización y rehabilitación se encontraban bien establecidas, por lo menos desde la ideología burocrática imperante (véase Azaola y Ruiz, 2009). De igual manera, es posible determinar que en ese esquema burocrático tanto la prevención delictiva como la participación ciudadana carecían de importancia (Pérez, 2004: 18-22; Piñeyro, 2004: 164).

No obstante, la prevención y la participación ciudadana se convirtieron en ejes centrales en la política criminal del sexenio del presidente Felipe Calderón. En efecto, en numerosas ocasiones Calderón enfatizó la importancia de la prevención del delito, así como la función que en su esquema preventivo le correspondía a la sociedad civil. Ante este escenario, resulta trascendental determinar los rasgos específicos bajo los cuales se convirtieron en interés gubernamental de primer orden. 
La hipótesis central de este trabajo es que el discurso político-preventivo en ese sexenio tuvo fundamentos gnoseológicos y éticos muy similares a los que han sido observados y teorizados en otros países, aunque con una diferencia significativa: mientras que en esos lugares la racionalidad preventiva es esencialmente comunitaria, en México fue familiar. En el presente trabajo no se pretende reseñar ni evaluar el desempeño de los programas de prevención puestos en práctica por el gobierno federal, sino - siguiendo a Miller y Rose (2008) - escrutar las racionalidades que, con o sin intención, subyacen en el diseño de esos programas, así como las representaciones que son movilizadas y enfatizadas a través de la construcción discursiva de la prevención.

La conexión entre política o "gobernanza", discurso y nociones criminológicas ha sido articulada con anterioridad - en mayor o menor medida por numerosos académicos (véase Scheingold, 1984; 1991; 1998; Sparks, 2000; Garland, 2001; Simon, 2007). La importancia de estas aproximaciones consiste en que suministran valiosas orientaciones teóricas y elementos empíricos sobre la influencia de los discursos y las narrativas políticas en el campo del control y prevención delictivos. No obstante, la mayoría de ellas están pensadas para funcionar en niveles abstractos de análisis. El análisis de discurso es más bien casuístico, llevado a cabo más con la finalidad de ilustrar algún punto particular que de efectuar un estudio sistemático.

En términos similares se han estudiado las políticas de control de narcotráfico en México, utilizando el discurso más como una técnica para esclarecer algunos aspectos que como objeto de interés sustantivo (véase Sandoval, 2000; Piñeyro, 2004; Astorga, 2007; Aguilar y Castañeda, 2009). ${ }^{1}$ Estos estudios, además, se han llevado a cabo más desde una perspectiva políticohistórica que criminológica y no han analizado la prevención delictiva, muy probablemente por la irrelevancia que este tema tuvo por tantos años.

La intención de este trabajo es superar estas limitaciones mediante el uso del estado actual de la criminología para la investigación metódica del discurso oficial sobre la prevención del delito en México. El estudio se divide en cuatro partes. En la primera se revisa someramente la literatura sobre el cambio de modelo criminológico mencionado. En la segunda se

1 El trabajo de Sergio García Ramírez (1989) incluye un voluminoso apéndice en el que se transcriben discursos, comparecencias, conferencias y entrevistas que concedió mientras fue procurador general de la República, todos relacionados con el tema del narcotráfico. Sin embargo, esos materiales no son analizados. Por lo tanto, este trabajo no es un antecedente directo del que aquí se presenta. 
describe la metodología empleada. En la tercera se exponen los resultados del análisis y en la última se discuten con mayor profundidad.

\section{La criminología y la prevención del delito actuales}

Muchas de las modificaciones que implicó la transformación criminológica de los últimos años se debieron a un desplazamiento en las explicaciones dominantes sobre la "delincuencia" y el "delincuente", desde un paradigma sociológico-positivista hacia uno neoclásico (Garland, 2001; Melossi, 2008). En términos del primero, el individuo que comete algún ilícito es visto como un "enfermo social". La principal función del sistema de justicia penal en general y específicamente del subsistema penitenciario, es la de rehabilitar al individuo para su reinserción en la sociedad. Y es ésta también la principal fuente de prevención. Las causas de la falta de adaptación de la persona tienen que buscarse en la sociedad misma, en su estructura y condiciones que impulsan a los individuos - en ocasiones casi de manera ineluctable - a la comisión de ilícitos (Garland, 2001: 27-48; O'Malley, 2010: 23-52). Es una criminología inspirada en buena medida por sociólogos como Durkheim (1993; 2006a; 2006b), Merton (1938; 1957), Sutherland (1949) y los pertenecientes a la Escuela de Chicago (véase Park, 1925; Shaw y McKay, 1942). El paradigma neoclásico, por el contrario, absuelve a la sociedad de los delitos cometidos por sus miembros y responsabiliza a éstos mediante la fórmula filosófica del "libre albedrío" (O'Malley, 2010: 23-52). En efecto, la llamada perspectiva de la "Elección Racional" señala que los delitos son conductas llevadas a cabo con el propósito de satisfacer ciertas cuestiones consideradas por el ofensor como necesidades, lo que implica un proceso de toma de decisiones, aunque sea limitado y rudimentario (Clarke, 1997: 9-10). El referente clásico aquí es Beccaria (2001).

Para Garland (1996; 2001), esta nueva racionalidad constituye una "criminología de uno mismo" según la cual el delincuente podría ser cualquiera de nosotros al que se le presente la oportunidad correcta. En la prevención, esta aproximación generalmente va de la mano de teorías situacionales y de actividades de rutina, basadas en la noción de que los delitos ocurren cuando coinciden tres elementos: un ofensor potencial, un objetivo deseado y la ausencia de guardianes capaces (Clarke, 1997: 11). La intención es prevenir el delito minimizando los riesgos de victimización mediante la generación de incentivos - positivos o negativos apropiados para dirigir la voluntad de los individuos hacia conductas socialmente aceptadas. 
Simultáneamente, Garland (1996; 2001) ha identificado una "criminología del otro". Esta racionalidad es "anti-moderna", pues pretende "defender y mantener el orden y la autoridad, el afianzamiento de estándares morales absolutos, y la reafirmación de la tradición y el sentido común" (Garland, 2001: 184), a través de la polarización social y la representación estereotípica de los "delincuentes" como "los otros" (Garland, 1996: 461). A su vez, esta criminología ha sido identificada con el "autoritarismo social" de la corriente política del "neo-conservadurismo" (O'Malley, 1999: 185). La opción preventiva aquí es la búsqueda de la incapacitación o eliminación "del otro" a través de la exclusión social.

Ambas tendencias pueden apreciarse claramente en los esquemas de prevención y seguridad comunitaria que se han multiplicado por el mundo occidental: se "devuelve" la responsabilidad a los individuos y a las comunidades tanto de la producción de los delitos como de su prevención, al mismo tiempo que se enfatiza la solidaridad mecánica ${ }^{2}$ (Durkheim, 1993; 2006b) de la comunidad, haciéndola más sensible a cualquier conducta diferente a la norma. Por un lado, el resultado es la "normalización" del delito; por el otro, la indignación moral ante la "anormalidad" que encarna el delito. O'Malley (1999) ha caracterizado esta esquizofrenia como "castigo contradictorio y volátil".

Un resultado esencial de esas averiguaciones ha sido el descubrimiento de los "llamamientos a la comunidad" como la justificación primordial de las políticas de control y prevención delictivos en los últimos años (Crawford, 1995; 1998; 1999; 2007; Lacey y Zedner, 1995; Edwards y Hughes, 2002). La comunidad es imaginada como un ente homogéneo, responsable y estructurado en torno a valores conservadores cuyo deterioro moral se asocia con el desorden social y por el contrario, su fortalecimiento con la eficaz prevención delictiva (Lacey y Zedner, 1995: 301). Esta racionalidad ha generado tal proliferación de asociaciones público-privadas que en esos países hablar de prevención del delito es casi equivalente a hablar de prevención comunitaria del delito.

2 Según Durkheim, (2006b) la "solidaridad mecánica" es típica de las sociedades primitivas y se caracteriza por ser el resultado de una conciencia colectiva única y fuerte, en extremo sensible a cualquier violación de las normas sociales. (63-70). 


\section{Metodología}

\section{El Análisis Crítico del Discurso (ACD)}

Existen varias perspectivas filosóficas y estrategias empíricas para el análisis de discursos. ${ }^{3}$ En este documento se utiliza una serie de metodologías conocidas comúnmente como Análisis Crítico del Discurso (ACD) (Fairclough, 1992; 1995; Fairclough y Wodak, 1997; Van Dijk, 2001; 2008: capítulo 4). Como lo señala Van Dijk (2001: 353-354), el ACD no tiene una estructura teórica unitaria, sino que se trata de un enfoque multidisciplinario que analiza la forma en la que el lenguaje - como una herramienta cultural - media las relaciones de poder que son instauradas, reproducidas y resistidas en interacciones sociales, instituciones y cuerpos de conocimiento (Van Dijk, 2001: 352; Rogers et al., 2005: 367). ${ }^{4}$

En este análisis la variable de "poder" ocupa un lugar preponderante. Según Wright Mills (1972: 40-41), la cuestión esencial sobre el poder es la fijación de los actores sociales que poseen capacidad de decidir o consecuentemente, no decidir. Llevado al terreno del discurso, el problema del poder se traduce en la capacidad de "producir conocimiento" - o en la falta de poder de ciertos actores sociales para generarlo- y las circunstancias bajo las cuales se produce (Cheek 2004). Es decir, se trata de considerar a las relaciones de poder como elementos discursivos (Fairclough y Wodak, 1997: 387-399).

Otra variable fundamental para el ACD es la de "'ideología"”, considerada como un conjunto de representaciones o construcciones de la realidad integrado a las formas y los significados de las prácticas discursivas cuya finalidad es mantener o transformar las relaciones de poder (Fairclough, 1992: 87). Las ideologías son más eficientes cuando se entremezclan en el discurso de tal forma que aparezcan como consideraciones de "sentido común", un proceso que Fairclough ha denominado la "naturalización" de las ideologías (87-88).

El análisis de los discursos presidenciales en México es relevante, precisamente, porque a pesar de la paulatina democratización del país, la figura presidencial mantiene una alta concentración de poder, no sólo constitucional y legal, sino sobre todo simbólico (Bourdieu, 1991), y por lo tanto su

3 Para listados no exhaustivos de los tipos de análisis discursivos existentes véase: Fairclough (1992: capítulo 1); Schiffrin, Tannen y Hamilton (2001); Wood y Kroger (2000: capítulo 2 y apéndice B).

4 Para un listado de los principios básicos del ACD véase Fairclough y Wodak (1997: 387-399). 
ocupante debe ser conceptualizado como un "definidor primario" (Hier et al., 2011: 267). Es por ello que los discursos presidenciales constituyen un instrumento esencial para producir significados - a partir de determinadas preconcepciones ideológicas - y transmitirlos a una audiencia, "problematizar" 5 la realidad y formular tecnologías de intervención destinadas a transformarla (Scheingold 1998; Cheek, 2004; Foucault, 2007; Miller y Rose, 2008). Pero además, como todo acto político, otra de las funciones de estos discursos es la de mantener y aumentar el poder presidencial a través del manejo ideológico de la concepción de la realidad. La naturaleza del ACD permite describir y analizar ambas finalidades del discurso presidencial, tanto la de "conocer" la realidad para modificarla, como la de mantener el status quo.

Por último, una cuestión que es importante aclarar es que los discursos presidenciales indican un "deseo de gobernar" de cierta manera (Miller y Rose, 2008: 71), mas no otorgan pistas suficientes para descifrar su poder constructivo en la realidad, ni las características del contexto receptivo en el que se producen (Van Dijk 2005: 58-76). Por lo tanto, esta investigación no pretende desentrañar el aspecto social de los discursos presidenciales, que comprende, por ejemplo, las condiciones de comprensión e internalización del discurso por parte de la audiencia, la interacción entre audiencia y emisor y la actitud de la audiencia hacia el mensaje (por ejemplo, de resistencia o adopción).

\section{Estrategia de recolección y análisis de datos}

Se revisaron los aproximadamente 1,400 discursos públicos del presidente Felipe Calderón pronunciados entre diciembre de 2006 y diciembre de 2009. ${ }^{6}$ Aquellos cuyo contenido está relacionado con la prevención del delito fueron revisados con mayor detenimiento. De esta manera se configuró una base de datos de 50 discursos. Además, se revisó el Plan Nacional de Desarrollo 2007-2012 (PND).

5 De acuerdo con Miller y Rose (2008: 14-16), “problematizar" se refiere al proceso a través del cual ciertas cuestiones e intereses son conceptualizados y construidos como problemas. Incluye no sólo la elaboración de los problemas en sí, sino también la especificidad que permite una intervención cuya finalidad sea la de transformar la realidad.

6 Todos los discursos del ex presidente Calderón pueden ser consultados en línea en la siguiente dirección: <http://www.presidencia.gob.mx/prensa/discursos/>. Para el presente trabajo se consultaron durante febrero de 2012. 
Esta estrategia metodológica condice con la propuesta hecha por Cheek (2004: 1145-1146), quien señala que el análisis de discurso utiliza técnicas "convencionales" de recolección de datos para producir textos que permitan estudiarlos desde cierta perspectiva del análisis de discurso y desde un paradigma teórico determinado. Éstos pueden incluir transcripciones de entrevistas, discursos públicos y documentos, entre otros.

El análisis de los datos se hizo a través de búsquedas sucesivas de información (Morse et al., 2002: 18). Los datos se interpretaron conforme se fueron recolectando y la información generada se utilizó como indicador para exploraciones posteriores. La intención fue crear un proceso continuo por virtud del cual los hallazgos parciales pudieran ser reforzados o refutados. Finalmente, se realizó una revisión comprensiva de la información bajo la perspectiva teórica reseñada.

\section{La construcción discursiva de la prevención del delito}

En la sección 1.11 del PND se señala que la función de la prevención del delito es la de "eliminar los problemas que puedan llevar a un joven a delinquir" y "evitar que haya más mexicanos que se conviertan en delincuentes, que sufran violación a su integridad y su patrimonio o que queden atrapados por el consumo de drogas". Desde aquí se pueden apreciar las preocupaciones de la administración. En este documento se tratarán principalmente dos de ellas: los jóvenes o niños y las drogas. La familia se agregará como un tercero. Como se verá, la construcción discursiva de la prevención se articula en torno a estos temas que han sido ampliamente desarrollados en el discurso oficial.

Los jóvenes y las drogas

La prevención del delito está destinada mayoritariamente a jóvenes y niños. ${ }^{7}$ La razón es que enfrentan un riesgo singular: las drogas. Considérese el siguiente extracto:

7 “[...] necesitamos al actor más importante en la lucha contra el crimen y contra la delincuencia que es el ciudadano y, particularmente, los jóvenes." Calderón, Felipe. El Presidente Calderón en la presentación del Programa Escuela Segura Tlaquepaque, Jalisco, México, 6 de febrero de 2007. 
Quienes hemos estado ya durante dos años y medio trabajando comprometidos contra el tráfico ilícito de drogas, hemos visto, y con tristeza, cómo son reclutados en todo el país, día con día, jóvenes, apenas adolescentes, incluso niños, que a través de las adicciones inducidas en sus personas, se vuelven literalmente esclavos de sus proveedores de drogas.

Hemos visto acciones de introducción de drogas e inducción a su uso en menores de siete u ocho años de edad con más frecuencia de lo que pudiéramos imaginar. Hemos visto jóvenes reclutados en esta esclavitud que, primero, la asumen gratuitamente con la curiosidad de la novedad, de la imitación, de la aceptación social en su grupo.

Posteriormente, la donación de drogas se termina y comienza una venta obligada, de sus proveedores; posteriormente, el niño, ya adolescente, empieza a abrir el monedero de su madre, posteriormente a robar los espejos de los vehículos de sus vecinos y se involucra con su grupo en actividades delictivas. ${ }^{8}$

El trinomio jóvenes/niños/drogas-delitos está presente en virtualmente todos los discursos sobre prevención del delito del presidente Calderon. En realidad es la narrativa ${ }^{9}$ oficial sobre la génesis delictiva. No existe teoría criminológica rival en cuanto a la extensión y detalle con que es formulada o la insistencia con que se plantea. ${ }^{10} \mathrm{La}$ cohesión y fuerza de los enunciados y los párrafos dan la idea de causalidad: si el joven o niño es "reclutado en esta esclavitud" de la adicción, entonces cometerá delitos. En efecto, el término posteriormente - utilizado en tres líneas consecutivas en el último párrafo - desplaza en el tiempo un evento inexorable. La conexión no es fortuita, sino sistemática. En el siguiente párrafo, por ejemplo, Calderón hace referencia a los mismos temas y en los mismos términos, sólo sustituyendo posteriormente por luego.

8 Calderón, Felipe. El Presidente Calderón conmemora el Día Internacional de la Lucha contra el Uso Indebido y el Tráfico Ilícito de Drogas. Ciudad de México, México, 26 de junio de 2009. (Cursivas añadidas.)

9 Las narrativas son herramientas para darle sentido a las cosas, y en muchas ocasiones codifican problemas y preocupaciones del agente, así como las soluciones que propone (Gee, 1999: 134).

10 Según Sacks (1996) una de las cuestiones más simbólicas sobre los discursos es precisamente la "insistencia con la que ciertas historias o explicaciones son realizadas" (59). 
Hoy la delincuencia busca generar esclavos entre los niños y jóvenes. Busca, primero, colocar droga, a veces de manera gratuita, generar una adicción, generar una dependencia en la escuela, en el barrio, y después cobrar esa droga que necesitará el joven; y ese joven empieza a hurgar en el monedero de la mamá, y luego a robar en los vehículos de los vecinos, y luego a encadenarse a una delincuencia que sirve, precisamente, a los amos que buscan esa nueva esclavitud en los jóvenes. ${ }^{11}$

Pero no se trata sólo de la construcción discursiva de una relación causal entre adicción y delincuencia, sino también del inicio de una "carrera criminal". Una vez cometido el primer ilícito, casi inocente (robar del monedero de la madre), el joven se ve envuelto en una espiral descontrolada de criminalidad que lo lleva hasta el crimen organizado. En palabras de Calderón:

He dicho, estoy plenamente convencido de que las adicciones son la esclavitud del siglo XXI, que es una esclavitud que se busca deliberadamente por quien tiene poder de distribución de droga, busca generar dependientes que ni siquiera en el mediano en el corto plazo tienen tal necesidad de droga que comienzan a abrir el monedero de la mamá, comienzan a robar en las casas o en los vehículos de los vecinos para pagar su adicción y, posteriormente, comienzan a tomar la ruta de la delincuencia que es piramidal, que empieza con delincuencia menor, robo de espejos de automóviles, sigue con asaltos y termina con delincuencia organizada, secuestros. ${ }^{12}$

En este contexto resulta muy apropiado el uso iterativo del término "esclavitud" como metáfora de la adicción a las drogas. ${ }^{13}$ Esta figura tiene un

11 Calderón, Felipe. Palabras de Felipe Calderón en la ceremonia de entrega del Premio Nacional de Juventud. Ciudad de México, México, 3 de octubre de 2008. (Cursivas añadidas.)

12 Calderón, Felipe. El Presidente Calderón en la inauguración del Tercer Foro contra la Delincuencia: A quién le toca prevenir el delito. Ciudad de México, México, 28 de octubre de 2008. Calderón utiliza otras metáforas, aunque no de forma tan insistente. Por ejemplo: “Esta estrategia está enfocada a dar un tratamiento integral a las personas de cualquier edad que desafortunadamente han caído en la prisión que significa el sufrir alguna adicción" Calderón, Felipe, El Presidente Calderón en la presentación del Programa Nacional contra las Adicciones. Tecomán, Colima, México, 17 de abril de 2007. "Por ello debemos actuar de inmediato, lo estamos haciendo, y haremos en estos seis años todo lo posible para salvarlos de las garras de las drogas y del peligro del crimen." Calderón, Felipe. El Presidente Calderón en el anuncio de la Estrategia Integral para la Prevención del Delito y Combate a la Delincuencia. Ciudad de México, México, 7 de marzo de 2007. (Cursivas añadidas.) 
doble propósito: por una parte, configura una enérgica condena moral; por la otra, justifica la adicción y le confiere un significado amoral al inicio de los jóvenes en las drogas. A la esclavitud no se ingresa voluntariamente; por el contrario, la persona es "esclavizada" por algún agente externo. De ahí la forma pasiva en que se utilizan verbos como: inducir, reclutar y generar. Es decir, la delincuencia busca activamente generar dependencia en los jóvenes, inducirlos y reclutarlos en la adicción a las drogas. En este caso, se trata de dos agentes externos que esclavizan: las drogas y los proveedores (léase narcotraficantes o delincuencia organizada).

Sin embargo, el mecanismo narrativo se invierte en cuanto la comisión de delitos inicia. El primer extracto transcrito arriba da muestra clara de este cambio. Los primeros dos párrafos se refieren a las adicciones, aquí los jóvenes participan pasivamente. En el tercer párrafo, no obstante, la exposición se vuelve activa. El adolescente es quien comienza "a abrir el monedero de su madre", "a robar los espejos de los vehículos de sus vecinos". Se asoma la "elección racional" del joven. Si no elige correctamente - diciendo "no" a las drogas o a cometer delitos - se le presenta como un riesgo o amenaza a la seguridad:

Y también lo vemos como un problema de seguridad, porque precisamente en la necesidad de obtener recursos para pagar la adicción, nuestras comunidades y nuestros pueblos ven crecientes fenómenos de delincuencia, por el lado de los dependientes. ${ }^{14}$

La causalidad en el discurso de Calderón se mantiene no porque en realidad piense que los jóvenes adictos a las drogas inevitablemente cometerán delitos cada vez más graves, sino porque esta especificidad discursiva le permite introducir cuestiones morales y criminalizar conductas consideradas indeseables, pero que no son ilegales. En teoría, consumir drogas no es un delito. La unión discursiva e inextricable entre consumo de drogas y delincuencia le permite ensanchar el derecho penal. ${ }^{15}$

14 Calderón, Felipe. El Presidente Calderón en la presentación del Programa Nacional contra las Adicciones. Tecomán, Colima, México, 17 de abril de 2007.

15 Lo mismo acontece con la introducción de los jóvenes a las drogas. Calderón reconoce que la voluntad de los jóvenes juega un papel en la ecuación de las adicciones. Es decir, aunque son "inducidos", al final de cuentas son ellos los que toman la decisión. Por eso mismo insiste en que los padres de familia, los maestros de escuelas, las organizaciones civiles (incluidas las iglesias), las empresas y las comunidades en general se involucren en la prevención de las adicciones concientizando a los niños y jóvenes sobre los peligros de las drogas. 
Pero la conducta es la única criminalizada. El discurso oficial desliga completamente el "qué" del "quién". Pese a que el joven adicto es representado como un peligro o un riesgo, el tono de la narración no cambia. Sigue siendo benevolente, compasivo, comprensivo. Los jóvenes siguen siendo "nuestros", siguen siendo los "hijos de sus padres", parte integral de la familia, que es "lo más valioso que tenemos los mexicanos". ${ }^{16}$ En efecto, el último párrafo transcrito es inmediatamente precedido en el discurso original por el siguiente:

En las últimas décadas hemos visto con mucha tristeza cómo se ha incrementado el número de nuestros jóvenes que transitan del alcohol o del tabaco, transitan al consumo de otras sustancias tóxicas. ${ }^{17}$

Líneas más arriba Calderón menciona:

Yo entiendo muy bien el dolor de un padre o de una madre cuando se entera de que su hijo ha caído en las garras de las drogas o del alcohol... Yo comprendo el sufrimiento de los padres, insisto, al ver cómo las adicciones vulneran a sus pequeños, a sus jóvenes, los alejan del hogar y los apartan del estudio. ${ }^{18}$

La moralización de la prevención no es un hecho que el discurso oficial pretenda ocultar. Por el contrario, lo afirma expresamente y lo ostenta como su motor y, en general, el de la guerra contra el crimen organizado. Según Calderón:

Nuestra causa es la más alta y del más elevado sentido moral, es preservar el futuro de México, es rescatar a los niños y a los jóvenes de las garras de la violencia, de la delincuencia y de las adicciones, es la seguridad de los mexicanos, es el futuro de la Nación. ${ }^{19}$

16 Calderón, Felipe. El Presidente Calderón en la presentación del Programa Escuela Segura. Tlaquepaque, Jalisco, México, 16 de febrero de 2007.

17 Calderón, Felipe. El Presidente Calderón en la presentación del Programa Nacional contra las Adicciones. Tecomán, Colima, México, 17 de abril de 2007.

18 Calderón, Felipe. El Presidente Calderón en la presentación del Programa Nacional contra las Adicciones. Tecomán, Colima, México, 17 de abril de 2007.

19 Calderón, Felipe. El Presidente Calderón en la ceremonia conmemorativa al CXLV Aniversario de la Batalla del 5 de mayo de 1862. Puebla, Puebla, México 5 de mayo de 2007. 
El problema es que el llamado a la moralidad no alcanza para movilizar, para crear consensos. Para eso hay que anclarlo en la vida diaria. Miller y Rose (2008:31) han señalado que el lenguaje desarrolla vínculos entre "los llamados éticos, epistemológicos y ontológicos del discurso político - a la nación, a la virtud, a lo que es o no es posible o deseable - y los planes, esquemas y objetivos que pretenden atender problematizaciones específicas en la existencia social, económica y personal". Es decir, una finalidad fundamental de los discursos es precisamente la de traducir ese sustento axiológico al lenguaje de la experiencia cotidiana, la de normalizarlo. En la cita inmediata anterior se observa la forma en que el discurso oficial transita constantemente entre ideas abstractas, como "preservar el futuro de México" y "la seguridad de los mexicanos", y su concretización en categorías relacionales como "rescatar a los niños y a los jóvenes de las garras de la violencia, de la delincuencia y de las adicciones". Es aquí donde resulta conveniente analizar la construcción discursiva de la familia.

\section{La familia}

La familia es el dispositivo discursivo-moral empleado para "enganchar" a la audiencia. Considérese la siguiente cita:

La inseguridad, la violencia, el narcotráfico, las adicciones son la amenaza más clara contra lo más valioso que tenemos los mexicanos, que es la familia y contra lo más valioso que tenemos los padres, que son los hijos. ${ }^{20}$

La inseguridad, la violencia, el narcotráfico y las adicciones son importantes para los ciudadanos, pero se trata de una importancia mediada por el efecto que puedan tener en la familia y, a través de ésta, en los hijos. Véase que los sujetos niños y jóvenes son constantemente utilizados en el contexto familiar, ya sea en referencia a los padres de familia o de forma más reveladora, como hijos. El discurso oficial centraliza su intervención sobre la familia porque la considera lo más preciado para la población, y en los jóvenes y niños como parte integral de ésta. La siguiente cita revela rotundamente la trascendencia de la familia:

20 Calderón, Felipe. El Presidente Calderón en la presentación del Programa Escuela Segura. Tlaquepaque, Jalisco, México, 6 de febrero de 2007. 
Estoy convencido de que la familia no sólo es el corazón de México, sino el corazón de toda sociedad; es la estructura que da sentido a la vida económica, política, social y cultural; es la base sobre la que se construye la identidad, los principios y los valores de las personas y la premisa básica para alcanzar un desarrollo humano sustentable, que definiera Paulo VI, como el paso de condiciones menos humanas a condiciones de vida cada vez más humanas. ${ }^{21}$

Calderón se refiere aquí a una versión idealizada de familia, aunque la disposición discursiva haga parecer que la narración es una mera representación fáctica. Es decir, las familias mexicanas - por el solo hecho de serlo - actualizan los más altos valores y principios, que posteriormente transfieren a sus miembros. La familia opera como una metonimia de esos valores: son una y la misma cosa. Hay dos excepciones:

En México más de cinco millones de familias están encabezadas por la madre, por una mujer. También presenciamos cada vez más que, de acuerdo con la legislación civil, la práctica de divorcio propicia que muchas familias vivan un proceso de desintegración y de reintegración, en ocasiones hacia nuevos núcleos familiares.

Este fenómeno es real y, aunque preocupante, es fundamental afrontarlo desde la perspectiva de los valores. Esta situación a lo que debe impulsarnos es a fortalecer, y fortalecer más que nunca, lazos familiares fundados en valores, sustentados en la confianza, en la lealtad, en el respeto recíproco, en la sinceridad y, sobre todo, en el amor. ${ }^{22}$

Las familias encabezadas por la madre o aquellas que experimentan un proceso de desintegración y reintegración debido a la práctica de divorcio ${ }^{23}$ no se acomodan en la categoría de familia. Estos fenómenos son preocupantes porque estas familias - por el solo hecho de la forma en que están constituidas - carecen de esos valores, o por lo menos no los tienen en la misma medida que las demás. Los valores familiares son imaginados exclusivamente en términos conservadores. En esa misma ocasión, Calderón

21 Calderón, Felipe. . El Presidente Calderón en el VI Encuentro Mundial de las Familias 2009. Ciudad de México, México, 14 de enero de 2009.

22 Calderón, Felipe. El Presidente Calderón en el VI Encuentro Mundial de las Familias 2009. Ciudad de México, México, 14 de enero de 2009.

23 Nótese que la práctica del divorcio es la que ocasiona la desintegración familiar y no al revés. 
menciona que Mexico es un país "con una firme tradición familiar". ${ }^{24}$ De la misma manera podría haber dicho que en México la familia es la familia tradicional.

De la "preocupación" gubernamental por estas familias incompletas deviene la necesidad de "fortalecer, y fortalecer más que nunca" los lazos familiares fundados en esos valores. ${ }^{25}$ La comunidad, las organizaciones sociales y el gobierno son el sucedáneo familiar:

Quienes tenemos la fortuna de pertenecer o de formar parte de una familia sólida estamos obligados a la solidaridad, a transmitir los valores que nos dan fuerza y nos identifican y a buscar la manera de compensar subsidiariamente desde la comunidad, desde las organizaciones sociales, desde las comunidades intermedias, desde las instituciones públicas y, por supuesto, desde el Gobierno a quienes no tuvieron esa oportunidad de vida de familia. ${ }^{26}$

Calderón se refiere aquí a subsanar la falta de valores familiares y no otras carencias. Regresando al tema de la juventud adicta a las drogas, la siguiente cita pone este punto de manifiesto con notable nitidez:

Una juventud que por sus condiciones sociales, familiares, educativas, por falta de oportunidades, tienen pocos asideros trascendentes, que tienen poco que creer, que no creen en la familia, que no tuvieron; que no creen en la economía ${ }^{27}$ o en la escuela, que no creen en Dios, porque no lo conocen. ${ }^{28}$

Calderón reconoce que la ausencia de oportunidades sociales, económicas y educativas influye en la drogadicción y la delincuencia. Sin embargo, no se trata de una preocupación discursiva. Entre todos los discursos

24 Calderón, Felipe. El Presidente Calderón en el VI Encuentro Mundial de las Familias 2009. Ciudad de México, México, 14 de enero de 2009.

25 "Tenemos que encontrar la manera de apoyar a quienes por diversas razones no forman parte de un núcleo familiar tradicional" Calderón, Felipe. El Presidente Calderón en el VI Encuentro Mundial de las Familias 2009. Ciudad de México, México, 14 de enero de 2009.

26 Calderón, Felipe. El Presidente Calderón en el VI Encuentro Mundial de las Familias 2009. Ciudad de México, México, 14 de enero de 2009.

27 Quizá no esté de más decir que la noción de "economía" se construye alrededor de las ideas básicas neo-liberales de libre mercado, eficiencia, competitividad, productividad e inversión privada (PND: parte 2).

28 Calderón, Felipe. El Presidente Calderón conmemora el Día Internacional de la Lucha contra el Uso Indebido y el Tráfico Ilícito de Drogas. Ciudad de México, México, 26 de junio de 2009. 
analizados, en sólo dos hace mención de ese factor, y en éstos toca el tema de forma superficial. Incluso, en uno de ellos más bien hace eco de la idea presentada por otro actor social. ${ }^{29}$ La razón de la irrelevancia de esta causa es que Calderón no la considera - en términos lógicos - suficiente. Esto es, al contrario de las fallas morales, no supone que la ausencia de oportunidades garantice la delincuencia o la drogadicción. Su efecto pernicioso es mediado a través de los valores familiares y religiosos, que a su vez favorecen la construcción de un 'tejido social' fuerte que pueda contener las tentaciones delictivas individuales. En sus palabras:

El crecimiento del crimen también se debe a un deterioro del tejido social, a una sociedad que tiene, en algunos casos, menor solidez en términos de valores y principios, de manera tal que pueda rechazar con contundencia la acción del crimen y la ilegalidad; una sociedad cada vez más vulnerable y con menor solidez en términos de valores que puedan defenderla de la acción de la delincuencia. ${ }^{30}$

De ahí la importancia de avanzar también en la regeneración del tejido social, en la impartición de valores desde la más temprana edad, en el fortalecimiento de las familias y de los lazos que unen a sus integrantes y a las familias entre sí... Por eso estoy convencido que para tener un México más seguro, para combatir las adicciones también es necesario, hoy más que nunca, fortalecer a la familia mexicana. ${ }^{31}$

La drogadicción y la delincuencia se problematizan en directa relación con la moralidad familiar porque al final de cuentas estos problemas sociales no recaen directamente en aquellas personas pobres, desaventajadas, sin educación ni habilidades sociales, sino en aquellas que teniendo estas características no creen en la familia - receptáculo de las creencias correctas

29 "Por otra parte, coincido plenamente con la doctora Morera que es indispensable no centrar la acción en contra del delito de la delincuencia en la parte persecutoria, como yo le llamaría, sino también enfatizar la parte preventiva y no podemos ser ajenos, omisos a las causas sociales y económicas que están también detrás del origen de la delincuencia o de la violencia" Calderón, Felipe. El Presidente Calderón en la inauguración del Tercer Foro contra la Delincuencia: A quién le toca prevenir el delito. Ciudad de México, México, 28 de octubre de 2010. (Cursivas añadidas.)

Calderón, Felipe. El Presidente Felipe Calderón, al término de la reunión que sostuvo con los organizadores de la marcha Iluminemos México. Ciudad de México, México 31 de agosto de 2008.

31 Calderón, Felipe. El Presidente Calderón en el VI Encuentro Mundial de las Familias 2009. Ciudad de México, México, 14 de enero de 2009. 
sobre la economía y Dios-y, por lo tanto, no "eligen" correctamente. Es esta falta de fe la que produce la criminalidad y las adicciones, la condición sine qua non de su existencia y prevalencia.

\section{Discusión}

El discurso oficial relativo a la prevención delictiva se caracteriza tanto por un continuismo como por una disociación. El primero se manifiesta en la conexión jóvenes-drogas-delitos; el segundo, en la falta de conexión entre los sujetos jóvenes y narcotraficantes. Los jóvenes son parte de nosotros; los narcotraficantes son los otros, vilipendiados constantemente en el discurso oficial como criminales, enemigos, y despreciables e indescriptibles seres. ${ }^{32}$

Estas categorías guardan pocas similitudes. Para la primera - como se vio - hay la construcción de una narrativa destinada a explicar las razones de su adicción y delincuencia; para la segunda no existe algo similar. Son seres que no califican como humanos, a los que - en palabras de un ex primer ministro británico - se debe "condenar más y entender menos" (citado en Garland, 2001: 184). Es cierto que en ambos casos existe una reprensión moral, pero sus alcances son muy distintos. En el primero el reproche no es a los individuos, sino a la acción (adicción, delincuencia). El discurso es incluyente, pues cualquiera de "nuestros" hijos podría "caer" en las adicciones o la delincuencia. Los jóvenes nunca son "drogadictos" o "criminales", aunque usen drogas y cometan delitos, e incluso, puedan llegar a pertenecer a la delincuencia organizada. ${ }^{33}$ Hay que diferenciarlos bien:

Y queremos distinguir en la ley, precisamente, entre los criminales que envenenan a los jóvenes y a los niños, y a quienes son sólo víctimas y padecen una enfermedad, una adicción que debe ser, precisamente, tratada como ello, como un problema de salud, más que como un problema criminal. ${ }^{34}$

32 Calderón, Felipe. El Presidente Calderón en la ceremonia de entrega de condecoraciones de la Policía Federal. Ciudad de México, México, 7 de agosto de 2009.

33 Véase, por ejemplo, los extractos de las páginas 11 y 12 de este texto, en los que Calderón hace referencia a las adicciones, delitos e involucramiento con la delincuencia organizada de los jóvenes sin usar esos epítetos.

34 Calderón, Felipe. Palabras de Felipe Calderón en la ceremonia de entrega del Premio Nacional de Juventud. Ciudad de México, México, 3 de octubre de 2008. 
En el segundo caso también se desaprueban vehementemente las acciones particulares, pero esta condena se deduce casi naturalmente del talante inmoral del individuo. Es la substanciación de la actividad delictiva en el sujeto "narcotraficante" o "criminal". La dislocación entre actor y acción es imposible en este caso, pues la conducta no es impersonal o abstracta. En otras palabras, no se le define como "persona que trafica drogas", sino como "narcotraficante". Y una vez colocada la etiqueta, la exclusión y estigmatización que esto acarrea facilita la atribución de una variedad casi incalculable de faltas - morales y legales, no importa - a su ethos malvado. Así, es el culpable del incremento en la criminalidad común, la decadencia moral y religiosa de la sociedad, el estancamiento económico y la disminución del turismo internacional, entre otros males. La consecuencia es que el riesgo que representan los jóvenes debe minimizarse, mientras que el que representan los narcotraficantes debe eliminarse..$^{35}$

Sobre la diferenciación entre ambos tipos de símbolos y del riesgo que personifican se elevan las "criminologías de uno mismo" y las "criminologías del otro". Las primeras apoyadas en la racionalidad liberal-económica de la "libertad responsable" y el manejo de riesgos (representados por los jóvenes); las segundas en la indignación moral y la expectativa irracional de la consecución de un "ambiente de cero-riesgo" (la eliminación de narcotraficantes y criminales) (Simon, 2007: 16). ${ }^{36}$

El hallazgo de la falta de estigmatización de los jóvenes resulta contraintuitivo tratándose del gobierno conservador de una sociedad también conservadora. ${ }^{37}$ La explicación debe buscarse en la construcción del "joven" como sinónimo de "hijo" y, más generalmente, en la "operacionalización" del edificio moral creado por el discurso oficial. Calderón construye los sujetos y objetos jóvenes (hijos), familia, narcotraficantes y drogas como símbolos de determinados valores o anti-valores. Estos símbolos son inteligibles, de sentido común, a diferencia de los valores, que son complejos

35 El argumento de este párrafo - y más indirectamente el de otros en este documento - invoca a los trabajos de Becker (1963) y Cohen (1972).

3636 Aunque las "criminologías de uno mismo" parecen ser moralmente neutrales - en contraposición con las "criminologías del otro" - debido a su carácter "científico", en realidad están basadas sobre concepciones de riesgo que implican una valoración moral y política, pues "un riesgo no es únicamente la probabilidad de un evento sino también la magnitud probable de su efecto, $y$ todo depende del valor que se le otorgue a ese efecto" (Douglas 1992b: 31). Y esta evaluación es, en el fondo, una cuestión "política, estética y moral" (31).

El descubrimiento del estigma asociado a los consumidores de droga es una tradición criminológica que continúa hasta nuestros días (véase Young, 1971; 2009; Cohen, 1972; Hathaway et al. 2011). 
y sutiles (Cohen, 1972: 41). Y son estos símbolos los que permiten la "normalización" de esa ideología conservadora en el discurso.

No obstante, esta construcción específica no atiende exclusivamente a consideraciones de tipo moral, sino también prácticas, es decir, políticas. Estas categorías constituyen los dos extremos de un proceso reforzado discursivamente cuyo propósito es unificar a la sociedad en torno a la representación idealizada de las familias - y consecuentemente de los hijos, sus jóvenes y niños - como víctimas (Simon, 2007: 75), y en contra de un enemigo común, lo que la galvaniza y la fustiga para actuar (Douglas, 1992a; 1992b). Al construir a las víctimas como inocentes e irreprochables, y al otro como perverso, más allá de cualquier tipo de redención, el discurso oficial apela a la indignación moral como resorte para la participación social. Calderón ha entendido - como muchos otros políticos destacados en el mundo - que en nuestros tiempos la movilización social es imprescindible para controlar y prevenir el delito, ${ }^{38}$ y no puede incitarla - por lo menos no a su favor - si aliena a los padres de familia atacando a sus hijos, "lo más valioso que tienen".

A diferencia de lo ocurrido en otros países, los "llamamientos a la familia" y no a la comunidad se convierten en el eje articulador del discurso preventivo. La comunidad juega un papel importante, pero primordialmente como un sustituto familiar o como una esfera de extensión de los valores familiares, equiparable a la función del gobierno y de las organizaciones no gubernamentales. Esto es así porque se asume que es en la familia - o, mejor dicho, en la falta de - donde se genera la delincuencia y la drogadicción en los jóvenes. Al respecto, Walker (1987: 187) menciona que una de las creencias simplistas y más ubicuas de los políticos de derecha es que "la mayor parte de las conductas delictivas es atribuible a la supervisión y crianza paternal laxa, a las actitudes paternales irresponsables y a la falta de censura para ver la televisión" y que "las medidas preventivas más eficaces están dirigidas al individuo". Son ciertamente parte de las "criminologías de uno mismo" que el discurso oficial aprueba. Otra de esas creencias, continúa Walker, es que la imposición de estigma - sin otorgar

38 Calderón hace constantes llamados a la participación ciudadana: “El México seguro que queremos para nuestros hijos requiere de la participación de la sociedad. Requiere de una participación responsable, pacífica, digna. Requiere de una participación que construya y no que destruya. Requiere del trabajo de los padres de familia, de las comunidades, para cuidar los espacios públicos, para denunciar el delito, para rechazar la ilegalidad y la corrupción". Calderón, Felipe. El presidente Calderón en la inauguración del edificio de seguridad pública del estado de Morelos y lanzamiento del programa Limpiemos México en la zona metropolitana de Cuernavaca. Temixco, Morelos, México, 27 de marzo de 2008. 
la posibilidad de reforma o rehabilitación - es una de esas medidas individuales de prevención (ibid:: 187). Aquí la similitud con las "criminologías del otro" son notables, pues el estigma es una condena moral que excluye al individuo del pacto moral colectivo, lo que acontece con las etiquetas de narcotraficante y criminal.

Se necesita más investigación para determinar las implicaciones prácticas de este hallazgo. Sin embargo, como se adelantó en el apartado anterior, es difícil que una construcción monolítica como la de "familia" en el discurso oficial preventivo pueda acomodar distintas concepciones, y una posible consecuencia es la exclusión de familias completas. En esta situación, sus hijos ya no cabrían en la categoría de jóvenes. De aquí al estigma de narcotraficante o criminal no hay mucha distancia.

\section{Bibliografía}

Aguilar, Rubén y Jorge Castañeda, 2009, El narco: la guerra fallida, México, Santillana.

Astorga, Luis, 2007, Seguridad, traficantes y militares: El poder y la sombra, México, Tusquets Editores.

Azaola, Elena y Ruiz, Miguel, 2009, “Política Criminal y Sistema Penal en México”, El Cotidiano, núm. 153, vol. 24, pp. 5-11.

Beccaria, Cesare, 2001, Tratado de los delitos y de las penas: precedida de una noticia sobre Beccaria, México, Porrúa.

Becker, Howard, 1963, Outsiders: Studies in the Sociology of Deviance, Londres, Free Press of Glencoe.

Berger, Peter y Thomas Luckmann, 1966, The Social Construction of Reality: A Treatise in the Sociology of Knowledge, Londres, Penguin Books.

Bottoms, Anthony, 1995, "The Philosophy and Politics of Punishment and Sentencing", en C. Clarkson y R. Morgan, eds., The Politics of Sentencing Reform, Oxford, Clarendon, pp. 17-50.

Bourdieu, Pierre, 1991, Language and Symbolic Power, Cambridge, Harvard University Press.

Cheek, Julianne, 2004, "At the Margins? Discourse Analysis and Qualitative Research", Qualitative Health Research, núm. 8, vol. 14, pp. 1140-1150. 
Clarke, Ronald, 1997 (ed.), Situational Crime Prevention: Successful Case Studies, Nueva York, Criminal Justice Press.

Cohen, Stanley, 1972, Folk Devils and Moral Panics, Londres, MacGibbon and Kee.

Crawford, Adam, 2007, "Crime Prevention and Community Safety", en M. Maguire, R. Morgan y R. Reiner, eds., The Oxford Handbook of Criminology (4ta. edición), Oxford, Oxford University Press, pp. 866-909.

Crawford, Adam, 1999, The Local Governance of Crime: Appeals to Community and Partnerships, Oxford, Oxford University Press.

Crawford, Adam, 1998, Crime Prevention and Community Safety: Politics, Policies and Practices, Londres, Longman.

Crawford, Adam, 1995, "Appeals to Community and Crime Prevention", Crime, Law, and Social Change, núm. 2, vol. 22, pp. 97-126.

Douglas, Mary, 1992a, "Risk and Blame”, en M. Douglas, ed., Risk and Blame: Essays in Cultural Theory, Londres, Routledge, pp. 3-21.

Douglas, Mary, 1992b, "Risk and Justice", en M. Douglas, ed., Risk and Blame: Essays in Cultural Theory, Londres, Routledge, pp. 22-37.

Durkheim, Émile, 2006a, El suicidio, México, Ediciones Coyoacán.

Durkheim, Émile, 2006b, Las reglas del método sociológico, México, Colofón.

Durkheim, Émile, 1993, La división del trabajo social, México, Colofón.

Edwards, Adam y Hughes, Gordon, 2002, "Introduction: The Community Governance of Crime Control", en G. Hughes y A. Edwards, eds., Crime Control and Community: The New Politics of Public Safety, Cullompton, Willan Publishing, pp. $1-19$.

Fairclough, N. y Wodak, Ruth, 1997, “Análisis Crítico del Discurso”, en T. van Dijk, ed., El Discurso como Interacción Social, México, Gedisa, pp. 367-404.

Fairclough, Norman, 1995, Critical Discourse Analysis: The Critical Study of Language, Nueva York, Longman.

Fairclough, Norman, 1992, Discourse and Social Change, Cambridge, Polity Press. 
Feeley, Malcolm y Simon, Jonathan, 1992, “The New Penology”, Criminology, núm. 4, vol. 30, pp. 449-474.

Foucault, Michel, 2007, La arqueología del saber, México, Siglo XXI Editores.

García, Sergio, 1989, Narcotráfico: un punto de vista mexicano, México, Porrúa.

Garland, David, 2001, The Culture of Control: Crime and Social Order in Contemporary Society, Oxford, Oxford University Press.

Garland, David, 1996, "The Limits of the Sovereign State: Strategies of Crime Control in Contemporary Societies", The British Journal of Criminology, núm. 4, vol. 36 , pp. 445-471.

Gee, James, 1999, An Introduction to Discourse Analysis: Theory and Method, Londres, Routledge.

Hathaway, Andrew, Comeau, Natalie y Erickson, Patricia, 2011, “Cannabis Normalization and Stigma: Contemporary Practices of Moral Regulation", Criminology and Criminal Justice, núm. 5, vol. 11, pp. 451-469.

Hier, Sean, Lett, Dan, Walby, Kevin y Smith, André, 2011, “Beyond Folk Devil Resistance: Linking Moral Panic and Moral Regulation", Criminology and Criminal Justice, núm. 3, vol. 11, Leeds, pp. 259-276.

Kemshall, Hazel, 2003, Understanding Risk in Criminal Justice, Maidenhead, Open University Press.

Lacey, Nicola y Zedner, Lucia, 1995, "Discourses of Community in Criminal Justice", Journal of Law and Society, núm. 3, vol. 22, pp. 301-325.

Melossi, Darío, 2008, Controlling Crime, Controlling Society: Thinking about Crime in Europe and America, Cambridge, Polity Press.

Merton, Robert, 1957, Social Theory and Social Structure, Glencoe, Free Press.

Merton, Robert, 1938, "Social Structure and Anomie", American Sociological Review, núm. 5, vol. 3, pp. 672-682.

Miller, Peter y Nikolas Rose, 2008, Governing the Present: Administering Economic, Social and Personal Life, Cambridge, Polity Press. 
Morse, Janice, Barrett, Michael, Mayan, Maria, Olson, Karin y Spiers, Jude, 2002, "Verification Strategies For Establishing Reliability and Validity in Qualitative Research", International Journal of Qualitative Methods, núm. 2, vol. 1, pp. 13-22.

O’Malley, Pat, 2010, Crime and Risk, Londres, Sage Publications.

O'Malley, Pat, 1999, "Volatile and Contradictory Punishment", Theoretical Criminology, núm. 2, vol. 3, pp. 175-196.

Park, Robert, 1925, "Community Organization and Juvenile Delinquency", en R. Park y R. Burgess, eds., The City, Chicago, University of Chicago Press, pp. 99112.

Pérez, Gabriela, 2004, Diagnóstico sobre la seguridad pública en México, México, Fundar, Centro de Análisis e Investigación, A.C.

Piñeyro, José, 2004, “Fuerzas Armadas y Combate a las Drogas en México: Ayer y Hoy", Sociológica, núm. 54, año 19, pp. 157-181.

Plan Nacional de Desarrollo 2007-2012 (PND), Estado de Derecho y seguridad, disponible en: <http://pnd.calderon.presidencia.gob.mx/eje1/prevencion-del-delito.

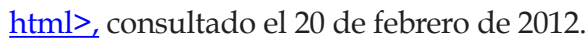

Reiner, Robert, 2006, “Beyond Risk: A Lament for Social Democratic Criminology", en T. Newburn and P. Rock, eds., en The Politics of Crime Control: Essays in Honour of David Downes, Oxford, Oxford University Press, pp. 7-49.

Rogers, Rebecca, Berkes, Elizabeth, Mosley, Melissa, Hui, Diane y O-Garro, Glynis, 2005, "A critical review of critical discourse analysis", Review of Research in Education, núm. 3, vol. 75, pp. 365-416.

Sacks, Valerie, 1996, "Women and AIDS: An Analysis of Media Representations", Social Science and Medicine, núm. 1, vol. 42, pp. 59-73.

Sandoval, Juan, 2000, "Militarización, Seguridad Nacional y Seguridad Pública en México", Espiral, Estudios sobre Estado y Sociedad, núm. 18, vol. 6, pp. 183-222.

Scheingold, Stuart, 1998, "Constructing the New Political Criminology: Power, Authority, and the Post-Liberal State", Law and Social Inquiry, núm. 4, vol. 23, pp. 857-895.

Scheingold, Stuart, 1991, The Politics of Street Crime: Criminal Process and Cultural Obsession, Filadelfia, Temple University Press. 
Scheingold, Stuart, 1984, The Politics of Law and Order: Street Crime and Public Policy, Nueva York, Longman.

Schiffrin, Deborah, Deborah Tannen, y Heidi Hamilton (eds.), 2001, The Handbook of Discourse Analysis, Oxford, Blackwell Publishers.

Shaw, Clifford y Henry McKay, 1942, Juvenile Delinquency and Urban Areas, Chicago, Chicago University Press.

Simon, Jonathan, 2007, Governing Through Crime: How the War on Crime Transformed American Democracy and Created a Culture of Fear, Nueva York, Oxford UniversityPress.

Sparks, Richard, 2000, "Perspectives on Risk and Penal Politics", en T. Hope y R. Sparks, eds., Crime, Risk and Insecurity, Londres, Routledge, pp. 129-145.

Sutherland, Edwin, 1949, White-collar Crime, Nueva York, Holt, Rinehart y Winston.

Van Dijk, Teun, 2008, Discourse and Power, Nueva York, Palgrave Macmillan.

Van Dijk, Teun, 2005, Estructuras y funciones del discurso, México, Siglo XXI Editores.

Van Dijk, Teun, 2001, “Critical Discourse Analysis", en D. Schiffrin, D. Tannen y H. Hamilton, eds., The Handbook of Discourse Analysis, Oxford, Blackwell Publishers, pp. 352-371.

Walker, Nigel, 1987, Crime and Criminology: A Critical Introduction, Oxford, Oxford University Press.

Wood, Linda y Rolf Kroger, 2000, Doing Discourse Analysis: Methods for Studying Action in Talk and Text, Thousand Oaks, Sage Publications.

Wright Mills, Charles, 1972, The Sociological Imagination, Nueva York, Oxford University Press.

Young, Jock, 2009, "Moral Panic: Its Origins in Resistance, Ressentiment and the Translation of Fantasy into Reality", The British Journal of Criminology, núm. 1, vol. 49, Londres, pp. 4-16.

Young, Jock, 1971, The Drugtakers, Londres, Paladin.

Recibido el 27 de abril de 2012 Aceptado el 20 de marzo de 2013 